\title{
A Combination of Chitosan, Cellulose, and Seaweed Polysaccharide Inhibits Postoperative Intra-abdominal Adhesion in Rats $^{\text {[ }}$
}

\author{
Lin Tian, Huan Li, Yan Li, Kun Liu, Yao Sun, Zhongcheng Cong, Xue Luan, Yao Li, \\ Jinglin Chen, Lin Wang, Zhihui Ren, Dengli Cong, Haotian Wang, and Jin Pei \\ School of Pharmaceutical Sciences, Jilin University, Changchun, Jilin, China
}

Received August 1, 2017; accepted December 7, 2017

\begin{abstract}
Intra-abdominal adhesion is a common complication after laparotomy. Conventional therapeutic strategies still cannot safely and effectively prevent this disorder. In this study, a combination of chitosan, cellulose, and seaweed polysaccharide (thereafter referred as CCS) was developed to significantly alleviate the formation of postoperative adhesion in rats with abdominal trauma. Transforming growth factor $\beta 1$ (TGF- $\beta 1$, an important promoter of fibrosis) and its downstream factors-namely, alpha-smooth muscle actin and plasminogen activator inhibitor-1 (PAI-1)were effectively suppressed by CCS in vivo, and as a result, the activation of tissue plasminogen activator (tPA, may generate plasmin that is a fibrinolytic factor capable of breaking down fibrin) was significantly promoted, presenting antifibrosis effects of CCS. In addition, the activity of kinases [e.g., transforming growth factor-activated kinase 1 (TAK1), c-Jun N-terminal kinase (JNK)/Stress-activated Protein Kinase (SAPK), and p38] in the mitogen-activated protein kinase (MAPK) inflammation signaling pathway was also significantly suppressed by CCS in vivo, demonstrating anti-inflammatory functions of CCS. The histologic studies further confirmed the role of CCS in the inhibition of fibrosis, collagen deposition, inflammation, and vascular proliferation. These results indicate the clinical potential of CCS in the treatment of postoperative intra-abdominal
\end{abstract}

adhesion. CCS may induce both antifibrosis and anti-inflammatory effects, potentially inhibiting the postoperative intra-abdominal adhesion. For antifibrosis effects, the expression of PAl-1 (a key factor for the adhesion formation) can be regulated by different TGF- $\beta 1$-associated signaling pathways, such as the Smads/p53 pathway, metalloproteinase/tissue inhibitor of matrix metalloproteinases pathway, Mitogen-activated Extracellular signal-regulated Kinase (MEK)/extracellular regulated protein kinase (ERK) pathway, and Yes-associated protein/ transcriptional coactivator with PDZ-binding motif pathway. Following the downregulation of PAI-1 achieved by CCS, the activation of tPA (which may generate plasmin that is a fibrinolytic factor capable of breaking down fibrin) is significantly promoted. For anti-inflammation effects, CCS may suppress the phosphorylation of classic kinases (e.g., TAK1, JNK, and p38) in the MAPK signaling pathway. In addition to the MAPK pathway, inflammatory pathways, such as Nuclear Factor- $\kappa$-gene Binding(NF- $\mathrm{kB}$ ), MEK/ERK, and Ras homologue protein/Rho associated coiled coil forming protein, are associated with the formation of intra-abdominal adhesion. Therefore, the prevention mechanisms of CCS will be further investigated in the future, with a hope of fully understanding of antiadhesion effects.

\section{Introduction}

Postoperative intra-abdominal adhesion is a common complication found in $90 \%-95 \%$ of patients undergoing abdominal surgery (Yang et al., 2012; Hu et al., 2015). The intra-abdominal adhesions do not only affect intestinal motility of patients, but they also cause abdominal pain, intestinal obstruction, intestinal necrosis, and other discomforts (Deng et al., 2016; Poehnert et al., 2016). Good surgical technique (e.g., the

This work was supported by the Jilin Province Development and Reform Commission (Grant 2014N149) and the Jilin Department of Science and Technology (Grant 20160209013YY).

https://doi.org/10.1124/jpet.117.244400.

S This article has supplemental material available at jpet.aspetjournals.org. avoidance of extensive surgical incisions and the use of microtraumatic/atraumatic suture materials) may minimize serosal injury and, therefore, decrease adhesion formation (Risberg, 1997; Liakakos et al., 2001). However, surgical technique alone cannot prevent de novo formation and, in particular, reformation of abdominal adhesions. Adjuvant therapeutic strategies to suppress the adhesion formation after peritoneal injuries comprise two categories. First, barriers (a medical implant that may reduce adhesions following surgery) are used to separate serosal surfaces during the early stages of wound repair and, therefore, inhibit coagulation, fibrin deposition, and fibroblastic activity. Barriers have been studied clinically to decrease the frequency and severity of postoperative adhesion formation (Parsak et al., 2007). Second, medicines are used to alter the

ABBREVIATIONS: CC, carboxymethyl chitosan; CCS, chitosan, cellulose, and seaweed polysaccharide; CL, cellulose; CS, chitosan; ECM, extracellular matrix; ELISA, enzyme-linked immunosorbent assay; ERK, extracellular regulated protein kinase; IL, interleukin; JNK, c-Jun N-terminal kinase; MAPK, mitogen-activated protein kinase; PAl-1, plasminogen activator inhibitor-1; qPCR, quantitative polymerase chain reaction; RT, room temperature; $\alpha$-SMA, $\alpha$-smooth muscle actin; SP, seaweed polysaccharide; TAK1, transforming growth factor-activated kinase 1 ; TGF- $\beta 1$, transforming growth factor $\beta 1$; TNF- $\alpha$, tumor necrosis factor- $\alpha$; tPA, tissue plasminogen activator. 
adhesion-induced inflammatory signaling pathways, suppressing the inflammatory process as a result (e.g., infection, endotoxin, and exudation) (Gomel et al., 1996). However, no treatment has been widely accepted to safely and effectively prevent postoperative intra-abdominal adhesions, and therefore, novel strategies are highly required.

Chitosan, the $N$-deacetylated derivative of chitin, has been used following intestinal surgery to promote tissue repair and avoid tissue adhesion (Francesko and Tzanov, 2011). Cellulose as a barrier can effectively separate the injured tissues, thus reducing the secretion of collagen and proliferation of fibroblast locally (Lou et al., 2012). Seaweed polysaccharides have been used as the adjuvant therapeutic material to reduce adhesion formation (Rocha et al., 2001). In this study, coadministration of chitosan, cellulose, and seaweed polysaccharide (we called the combination CCS) was performed in rats with abdominal surgery, and as a result, CCS significantly alleviated the intra-abdominal adhesion when compared with treatment using chitosan (CS), cellulose (CL), and carboxymethyl chitosan (CC, a derivative of chitosan widely used as an antiadhesion agent). In addition, the antifibrosis and anti-inflammation mechanisms of CCS were also confirmed in vivo. Consequently, CCS holds great therapeutic potential in the treatment of postoperative intra-abdominal adhesion.

\section{Materials and Methods}

Animals. Male Wistar rats (220-250 g) were used in this study. Animals were housed under standard laboratory conditions at a constant room temperature (RT) for at least 7 days before experiments. Rats were deprived of food for 12 hours but had free access to water before experiments. Animal works were reviewed and approved by the Animal Care and Use Committee of the Jilin University.

Reagents. CS, CL, seaweed polysaccharides (SPs), and CC were purchased from Sinopharm Pharmaceutical Co., Ltd. (Beijing, China). As described in the manufacturer's instructions, the polymeric level of CS was 2000-2100 with 70\% deacetylation, the degree of polymerization of CL was 215-240, and the polymerization degree of seaweed polysaccharide (it is extracted from brown seaweed) was 500. The aforementioned materials were directly used as advised by the suppliers. In addition, the other reagents were purchased from Sigma-Aldrich (St. Louis, MO) unless specially mentioned.

Five milligrams of CS, $3 \mathrm{mg}$ of CL, and $1.5 \mathrm{mg}$ of SP were dissolved in $1 \mathrm{ml}$ of sterile phosphate-buffered saline to prepare CCS. In addition, CS $(10 \mathrm{mg} / \mathrm{ml})$ and CL $(6 \mathrm{mg} / \mathrm{ml})$ were prepared for antiadhesion studies. It is worth noting that SP as an adjuvant material can increase the viscosity of CCS, but the effect of this component alone on the prevention of postoperative adhesion was mild (Rocha et al., 2001). Therefore, SP alone was not used in this work. In addition, $\mathrm{CC}$ is a nontoxic derivative of chitosan generated by carboxymethylation reaction $(70 \%)(\mathrm{Mn}=3 \mathrm{KD})$, and has been widely investigated in the treatment of intra-abdominal adhesion (Ryan and Sax, 1995). Therefore, CC ( $8 \mathrm{mg} / \mathrm{ml}$, phosphate-buffered saline) was used as the positive control in this study.

Experimental Design and Operative Procedure. Animals were anesthetized by intraperitoneal injection with chloral hydrate (10\%) at a concentration of $0.3 \mathrm{ml}$ per $100 \mathrm{~g}$ of body weight. Rats with intra-abdominal adhesion (the model group) were prepared as described by Zhang et al. (2014). In brief, the cavum abdominis was accessed, followed by a 2-3-cm abdominal midline incision, and the pouch-like cecum was exposed to the air for approximately 5 minutes. The cecum wall and its opposite parietal peritoneum were abraded mildly by the scalpel blade until the punctate hemorrhage was observed. In addition, the untreated group (without surgery) and the sham group (only with abdominal midline incision) were also prepared.
The doses of the compounds and their combination used in this study were first determined using healthy Wistar rats. Four hours following intraperitoneal injection of the compounds and their combination, interferon- $\alpha$, interleukin-6 (IL-6), and IL-12 in the serum were analyzed using enzyme-linked immunosorbent assay (ELISA) kits (Nanjing Jiancheng Bioengineering Institute, Nanjing, Jiangsu, China) according to the manufacturer's instructions. No acute immunologic response was caused using the doses mentioned here (data not shown).

At the time of surgery, the model group was treated using CCS with low, middle, and high doses (milligrams compound per kilogram of body weight):

Low dose: $20 \mathrm{mg} / \mathrm{kg} \mathrm{CS}+12 \mathrm{mg} / \mathrm{kg} \mathrm{CL}+9 \mathrm{mg} / \mathrm{kg} \mathrm{SP}$

Middle dose: $40 \mathrm{mg} / \mathrm{kg} \mathrm{CS}+24 \mathrm{mg} / \mathrm{kg} \mathrm{CL}+12 \mathrm{mg} / \mathrm{kg} \mathrm{SP}$

High dose: $60 \mathrm{mg} / \mathrm{kg} \mathrm{CS}+36 \mathrm{mg} / \mathrm{kg} \mathrm{CL}+18 \mathrm{mg} / \mathrm{kg} \mathrm{SP}$.

In addition, $2 \mathrm{ml}$ of saline, $80 \mathrm{mg} / \mathrm{kg} \mathrm{CS}, 48 \mathrm{mg} / \mathrm{kg} \mathrm{CL}$, and $32 \mathrm{mg} / \mathrm{kg}$ $\mathrm{CC}$ were administered into the abdominal cavity of the model group.

Animals were maintained for 14 days, and body weight was recorded regularly to further determine the toxicity.

Adhesion Grade and Assessment. On day 14 following the surgery, animals were anesthetized by intraperitoneal injection with chloral hydrate $(10 \%)$ at a concentration of $0.3 \mathrm{ml} / 100 \mathrm{~g}$ body weight. The abdominal wall was opened with an inverted "U"-shape incision. The adhesion was evaluated by the adhesion grade criteria reported by Nair et al. (1974) as follows: 0, complete absence of adhesion; 1 , single band of adhesion, between viscera or from viscera to abdominal wall; 2 , two bands, either between viscera or from viscera to abdominal wall; 3 , more than two bands, between viscera or viscera to abdominal wall or whole intestines forming a mass without being adherent to abdominal wall; 4, viscera directly adherent to abdominal wall, irrespective of number and extent of adhesive bands. The researchers who assessed the grade were independent and were blinded to the design.

ELISA. Blood was collected from the animal eyeballs, coagulated for 20 minutes at RT, and centrifuged at $3000 \mathrm{rpm}$ for 10 minutes. The serum tumor necrosis factor- $\alpha$ (TNF- $\alpha$ ), transforming growth factor $\beta 1$ (TGF- $\beta 1$ ), tissue plasminogen activator (tPA), and plasminogen activator inhibitor-1 (PAI-1) were evaluated using ELISA kits (Nanjing Jiancheng Bioengineering Institute) according to the manufacturer's instructions.

Western Blot. Total protein was extracted from the cecum and its opposite parietal peritoneum using Radio Immunoprecipitation Assay Lysis Buffer(RIPA) buffer [50 mM Trish- $\mathrm{HCl}(\mathrm{pH}=7.4), 150 \mathrm{mM} \mathrm{NaCl}$, $1 \%$ Triton-100, $1 \%$ sodium deoxycholate, $0.1 \%$ SDS, $1 \mathrm{mM}$ sodium orthovanadate, $1 \mathrm{mM}$ sodium fluoride, $1 \mathrm{mM}$ EDTA] containing $1 \mathrm{mM}$ phenylmethanesulfonyl fluoride (Beyotime, Shanghai, China) (Guo et al., 2017) .Protein concentrations were measured using the BCA Protein Assay kit (GenStar, Beijing, China). Twenty micrograms of proteins were loaded into the $12 \%$ SDS-PAGE gel and electrophoresed at $80 \mathrm{~V}$ for 30 minutes. Protein was then transferred to Polyvinylidene Fluoride(PVDF) membranes at $120 \mathrm{~V}$ for 80 minutes. The membranes were blocked with $5 \%$ bovine serum albumin, and then incubated with antibodies [anti-transforming growth factor-activated kinase 1 (antiTAK1) antibody, ab109526, 1:2000 (Abcam, Cambridge, UK); anti-cJun N-terminal kinase (anti-JNK)/phosphorylated JNK antibodies, ab17946/ab4821, 1:2000 (Abcam); anti-extracellular regulated protein kinase (anti-ERK)/phosphorylated ERK antibodies, ab196883/ab214362, 1:2000/1:1000 (Abcam); anti-P38/phosphorylated P38 antibodies, ab170099/ab4822, 1:2000/1:1000 (Abcam); anti- $\beta$-actin antibody, ab8226, 1:2000 (Abcam); anti-phosphorylated TAK1 antibody, ENP0424, 1:1000 (Elabscience Biotechnology, Wuhan, China); anti- $\alpha$-smooth muscle actin (anti- $\alpha$-SMA) antibody, ENT5053 Cat.ENT5053, 1:1000 (Elabscience Biotechnology)] overnight. Antibody reactive bands were detected using a bio-imaging system (Micro Chemi 4.2, DNR Bio-Imaging Systems Ltd., Jerusalem, Israel). Densitometry analysis of bands was performed using ImageJ (National Institutes of Health, Bethesda, $\mathrm{MD}$ ), and all results were normalized to $\beta$-actin control. 
Real-Time Quantitative Polymerase Chain Reaction. Total RNA was collected from the cecum and its opposite parietal peritoneum using the TransZol Up Reagent (ET111-01; TransGen Biotech, Beijing, China). First-strand cDNA was generated using the TransScript All-in-One First-Strand cDNA Synthesis SuperMix for qPCR Kit (AT341; TransGen Biotech). Gene expression was evaluated by real-time quantitative polymerase chain reaction (qPCR) with the TransStart Top Green qPCR SuperMix Kit (AQ131; TransGen Biotech) using the Applied Biosystems (Foster City, CA) Real Time PCR System (Step One Plus). The PCR reactions were carried out by 40 cycles of denaturation at $94^{\circ} \mathrm{C}$ for 5 seconds and annealing at $60^{\circ} \mathrm{C}$ for 30 seconds. The quantitative level of each target mRNA was measured as a fluorescent signal corrected according to the signal for glyceraldehyde-3-phosphate dehydrogenase mRNA. The $2^{-\triangle \triangle} \mathrm{Ct}$ method was used to quantify the relative changes in mRNA (Guo et al., 2012).

The PCR primer sequences were as follows: TGF- $\beta 1,5^{\prime}$-CAT TGC TGT CCC GTG CAG A-3' (forward) and 5'-AGG TAA CGC CAG GAA TTG TTG CTA-3' (reverse); TNF- $\alpha, 5^{\prime}$-AGG AGG TGC TCT CCG AGA AA-3' (forward) and 5'-TGA GGG CAT TGG CAT ACG AG-3' (reverse); tPA, 5' -CGC TGT ACC TCA CAG CAT CTG TTT A-3' (forward) and 5'-CAT CCG CTT ATC GAT CAT GCA C-3' (reverse); PAI-1, 5'-ACC ATC TCC GTG CCC ATG A-3' (forward) and 5'- GGG CAG TTC CAG GAT GTC GTA-3' (reverse); glyceraldehyde-3-phosphate dehydrogenase, 5' -ACC ACA GTC CAT GCC ATC AC-3' (forward) and 5' - TCC ACC ACC CTG TTG CTG TA-3' (reverse).

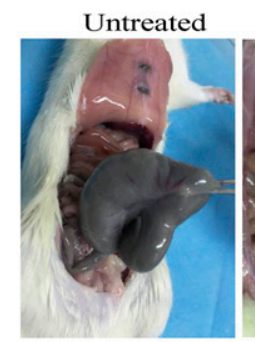

Model+CS
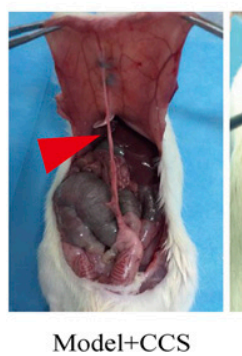

(Low dose)
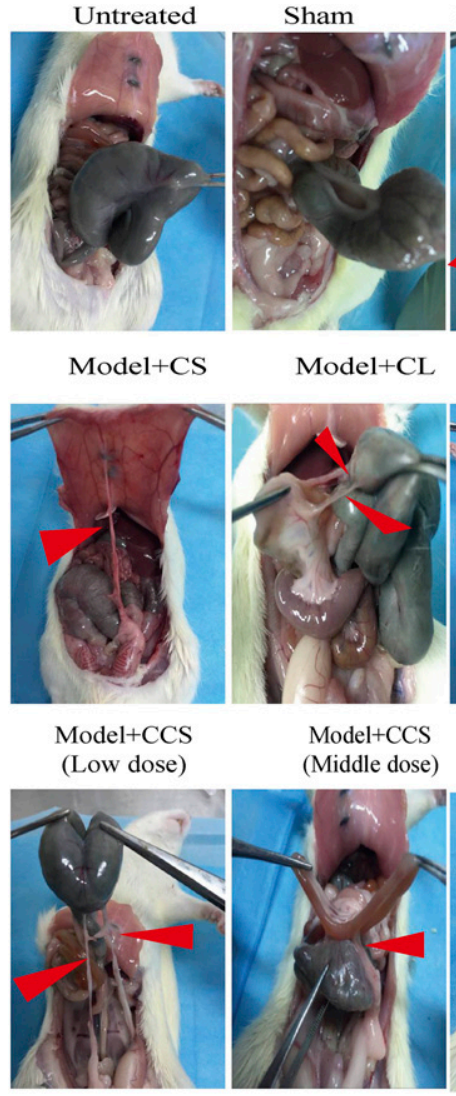

Model+CL

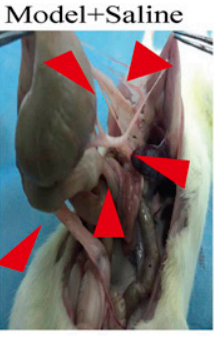

L Model+CC

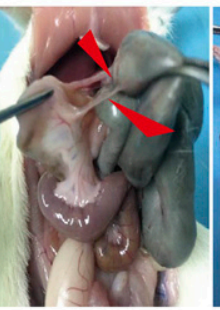

Model+CCS (Middle dose)

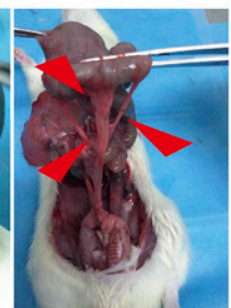

Model+CCS (High dose)

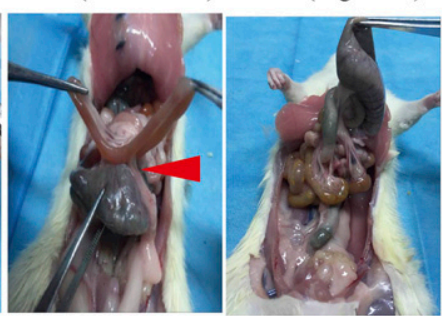

Fig. 1. Intra-abdominal adhesions (red triangle) in rats on day 14 after surgery $(n=10)$. No adhesion was observed in the untreated and sham groups, in which the surfaces of the parietal peritoneum and cecum were smooth. Obvious adhesions were formed in the model group treated with saline. Adhesions in the CS $(10 \mathrm{mg} / \mathrm{ml})$ and CL $(6 \mathrm{mg} / \mathrm{ml})$ groups were clearly less than in the model group and the CC group $(8 \mathrm{mg} / \mathrm{ml})$. CCS at high dose achieved effective antiadhesion results compared with CCS at low and middle doses.
Histologic Studies. Tissues from the surgical areas were fixed with $4 \%$ paraformaldehyde at RT; dehydrated in $70 \%, 80 \%, 90 \%$, and $100 \%$ ethanol $(\mathrm{v} / \mathrm{v})$; cleaned in xylene; and embedded in paraffin. Tissue sections $(5 \mu \mathrm{m})$ were stained using H\&E to assess inflammation and vascular proliferation. In addition, Masson Trichrome kits (G1340; Solarbio Science \& Technology, Beijing, China) and Picrosirius Red kits (G1470; Solarbio Science \& Technology) were used to assess the collagen deposition. Data were analyzed using the image acquisition and analysis system (Olympus Imaging, Tokyo, Japan).

Tissue sections $(5 \mu \mathrm{m})$ following the antigen restoration were also incubated with $3 \%$ hydrogen peroxide $\left(\mathrm{H}_{2} \mathrm{O}_{2}\right)$ solution at RT for 10 minutes, followed by incubation with TGF- $\beta$ 1antibody (ab25121; Abcam) at $4^{\circ} \mathrm{C}$ overnight. Tissue sections were then incubated 15 minutes within the MaxVision reagent (Maxim, Fuzhou, China) for visualization, and hematoxylin was used as the counterstain. Data were analyzed using the image acquisition and analysis system (Olympus Imaging).

Statistical Analysis. The SPSS 15.0 software (SPSS, Chicago, IL) was used for statistical analysis. Adhesion scores between different groups were analyzed using the Kruskal-Wallis H test and the MannWhitney $U$ test. Data are expressed as the median.

In addition, one-way analysis of variance was applied to test the significance of differences in three or more groups using Prism version 5 (GraphPad Software Inc., San Diego, CA). Data are expressed as the mean \pm S.D. In all cases, the significance level was set at $\mathrm{P}<0.05$.

\section{Results}

CCS Suppressed the Intra-abdominal Adhesion in the Rat Model. The antiadhesion effects of CCS were evaluated using Nair's scoring system in rats with abdominal surgery $(n=10)$ (Nair et al., 1974). On day 14 following the surgery, results from Fig. 1 and Table 1 showed that no obvious abdominal adhesion was found in the untreated group (score $=0$ ) and the sham group (score $=0$ ). In contrast, filamentary adhesions (indicated by red triangles) were clearly found in the model group treated with saline (score $=4$ ), which was similar to data from the model group without any treatment (data not shown; the results of the model group without any treatment were not shown for the following experiments, due to the similarity to the model group with saline treatment). When compared with the model group treated with saline, CCS at low dose (score $=2.5$ ) and middle dose ( significantly $(P<0.05)$ reduced the adhesion formation, which was similar to data achieved by CS (score $=2.5)$ and CL $($ score $=2$ ) but significantly better than results obtained by CC

\section{TABLE 1}

Scores of abdominal adhesion on day 14 after surgery

Median of adhesion scores represent the severity of adhesion in the untreated group, sham group, and the model groups treated with CS $(10 \mathrm{mg} / \mathrm{ml}), C L(6 \mathrm{mg} / \mathrm{ml})$, CC $(8 \mathrm{mg} / \mathrm{ml})$, and CCS at three doses $(n=10)$. Comparisons between different groups were applied using the Kruskal-Wallis $\mathrm{H}$ and Mann-Whitney $U$ tests (SPSS 15.0).

\begin{tabular}{lcccccc}
\hline \multirow{2}{*}{ Groups $(n=10)$} & \multicolumn{5}{c}{ Adhesion Score } & \multirow{2}{*}{ Median Score } \\
\cline { 2 - 6 } & 0 & I & II & III & IV & \\
\hline Untreated & 10 & 0 & 0 & 0 & 0 & 0 \\
Sham & 10 & 0 & 0 & 0 & 0 & 0 \\
Model + saline & 0 & 0 & 1 & 1 & 8 & 4 \\
Model + CS & 0 & 1 & 4 & 3 & 2 & $2.5^{*}$ \\
Model + CL & 1 & 1 & 3 & 2 & 3 & $2.5^{*}$ \\
Model + CC & 0 & 2 & 2 & 3 & 3 & 3 \\
Model + CCS (low dose) & 1 & 1 & 3 & 3 & 2 & $2.5^{*}$ \\
Model + CCS (middle dose) & 2 & 2 & 2 & 1 & 3 & $2^{*}$ \\
Model + CCS (high dose) & 2 & 1 & 3 & 4 & 0 & $2^{*} \#$ \\
\hline
\end{tabular}

$* P<0.05$, compared with the model group (saline).

${ }^{\#} P<0.05$, compared with CS, CL, and CC. 


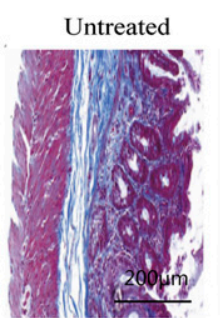

Model+CS

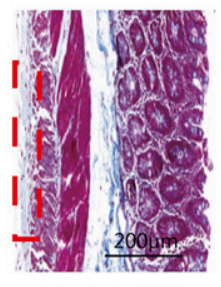

Model+CCS (Low dose)
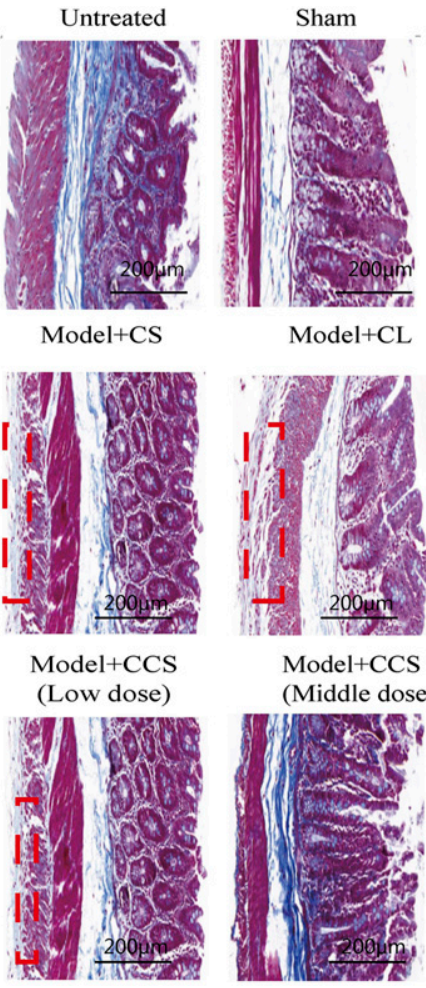

Model+CL

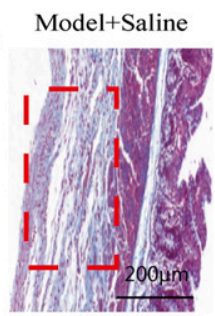

Model+ CC

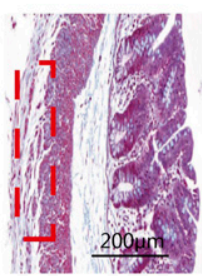

Model+CCS (Middle dose)

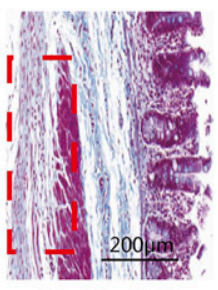

Model+CCS
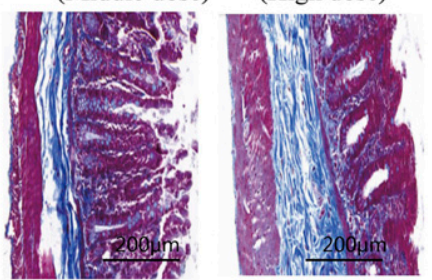

Fig. 2. The collagen deposition and fibrosis were demonstrated using Masson Trichrome staining (red tetragonum) in tissues with adhesion ( $n=$ 4) (original magnification, $100 \times$; scale bar, $200 \mu \mathrm{m}$ ). Collagen fibers and mucosa: blue; muscle fiber and cellulose: red.

(score $=3$ ). It is worth noting that CCS at high dose achieved a significant improvement (score $=2, P<0.01$ ) when compared with CCS at low and middle doses, and demonstrated a significantly better therapeutic effect $(P<0.05)$ than $\mathrm{CS}$, $\mathrm{CL}$, and CC. The results showed that CCS at a high dose was able to effectively prevent intra-abdominal adhesion formation in rats.

The Antiadhesion Properties of CCS Were Associated with Reduction of Collagen Disposition, Inflammation, and Angiogenesis. The formation of external collagen fibrils in the cecum serosa was detected using Masson Trichrome kits (Wei et al., 2017) (Fig. 2). In Fig. 2, the level of external collagen fibers (stained in blue within the red square) in the model group treated with saline was remarkably higher than the untreated and sham groups (no obvious blue staining). Although CS, CL, and CC presented the formation of collagen fibers (stained in blue within the red square), the levels of collagen disposition were significantly less than that of the model group treated with saline (Fig. 2). In addition, CCS at various doses significantly reduced the formation of collagen fibers, and more importantly, no obvious blue staining was observed in CCS at middle and high doses, demonstrating a better therapeutic effect when compared with CS, CL, and CC (Fig. 2).

The formation of collagen fibrils in the cecum serosa was further evaluated using Picrosirius Red kits (Bi et al., 2017) (Fig. 3). The external collagen fibers in the injured peritoneum were stained in red as indicated within the black square. Results from Fig. 3 are similar to those obtained using Masson Trichrome kits (Fig. 2), further confirming the prevention
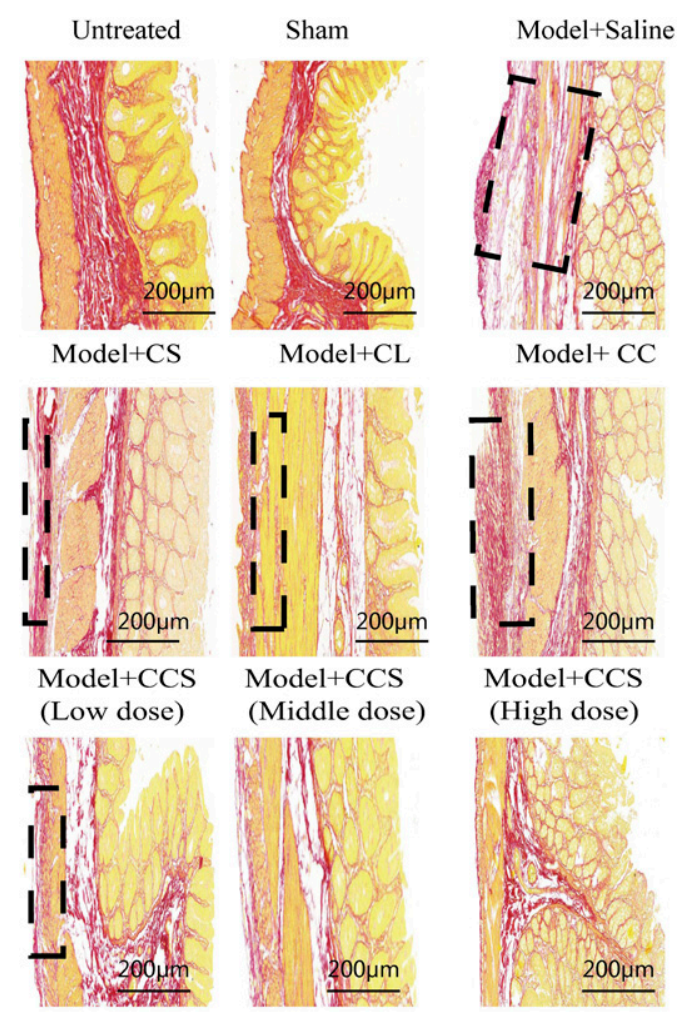

Fig. 3. The fibrosis in the intra-abdominal adhesions was demonstrated using Picrosirius Red staining (black tetragonum) $(n=4)$ (original magnification, $100 \times$; scale bar, $200 \mu \mathrm{m}$ ).

effect of CCS on adhesion formation, particularly at middle and high doses.

In addition, the inflammation and angiogenesis within the cecum serosa were assessed in terms of tissue morphology using H\&E staining (Fig. 4). In comparison with the untreated and sham groups, the cecum serosa was clearly damaged in the model group treated with saline due to the identification of plasma cells, granulocytes, and macrophages (shown in black squares) (Fig. 4), which was similar to results reported by Zhang et al. (2014). In addition, CS, CL, CC, and CCS (low dose) did not inhibit the induction of inflammation (shown in black squares). In contrast, CCS at middle and high doses significantly inhibited the inflammatory responses, which was similar to results found in the untreated and sham groups (Fig. 4). In addition, vascular proliferation was also observed with CC (indicated by red circles), which was similar to the model group treated with saline (Fig. 4).

Results from Figs. 2-4 indicated that the antiadhesion properties of CCS were associated with a reduction of collagen disposition, inflammation, and angiogenesis.

CCS Suppressed the Production of TGF- $\beta 1, \alpha$-SMA, and PAI-1 and Enhanced the Activation of tPA. TGF- $\beta 1$ is known to promote tissue fibrosis (Holmdahl et al., 2001). The mRNA level of TGF- $\beta 1$ in rat cecum was first assessed using real-time qPCR (Fig. 5A). As shown in Fig. 5A, the TGF- $\beta 1$ mRNA was significantly increased ( 7 -fold) in the model group treated with saline compared with the untreated and sham groups. Although CCS at various doses could not fully inhibit the expression of TGF- $\beta 1$ mRNA ( 2 -fold), it significantly decreased the level of TGF- $\beta 1$ mRNA compared with CS, CL, and CC (no less than 4-fold) (Fig. 5A). In addition, 


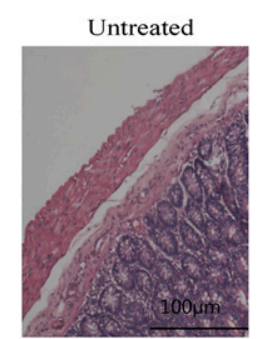

Model+CS

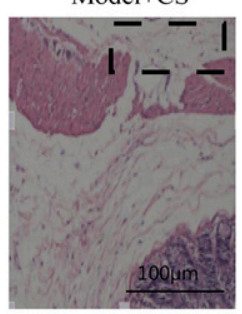

Model+CCS

(Low dose)

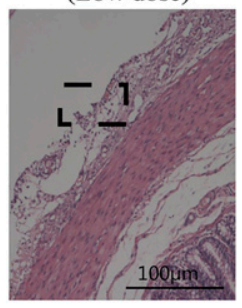

Fig. 4. Hematoxylin-eosin staining of injured tissues $(n=4)$. Black tetragonum represents the accumulation of plasma cells, granulocytes, and macrophages. Red circle represents the vascular proliferation and congestion (original magnification, $200 \times$; scale bar, $100 \mu \mathrm{m}$ ).

the protein level of TGF- $\beta 1$ was also assessed using ELISA (Fig. 5B). Interestingly, the expression of TGF- $\beta 1$ protein achieved by CCS at three doses was similar to that in the untreated and sham groups, but was significantly lower than that in the CS, CL, and CC groups (Fig. 5B). The expression of TGF- $\beta 1$ was also evaluated using immunohistochemical staining (Fig. 6). TGF- $\beta 1$ protein was clearly stained (indicated in red squares) in the CS, CL, and CC groups, but to less extent compared with the model treated with saline. In contrast, CCS at three doses significantly inhibited the TGF- $\beta 1$ expression, which was similar to data found in the untreated and sham groups (Fig. 6, indicated by red arrows). These results showed that CCS could significantly suppress the production of TGF- $\beta 1$ in an injured cecum.

The expression of two TGF- $\beta 1$ downstream factors, fibroblast marker $\alpha$-SMA (Fig. 7) and PAI-1 (Fig. 8), was also assessed in the injured cecum using real-time qPCR, Western blot, and ELISA. Results in Fig. 7 demonstrated that CCS at three doses significantly reduced the protein level of $\alpha$-SMA ( 1-fold), demonstrating a better inhibitory effect compared with CS, CL, and CC ( $\sim 2$-fold). In addition, the mRNA and protein levels of PAI-1 in the three CCS groups were also significantly reduced compared with results found in the CS, CL, and CC groups (Fig. 8, A and B). These results demonstrated that CCS was also able to significantly suppress the production of TGF- $\beta 1$ downstream factors in the injured peritoneum.

tPA (which can generate plasmin to break down collagen fibers) is negatively regulated by PAI-1 (Yang et al., 2008). Following the downregulation of PAI-1 (Fig. 8, A and B), as a result, the expression of tPA was significantly enhanced by CCS at various doses (Fig. 8, C and D), and more importantly, CCS (compared with CS, CL, and CC) demonstrated better tPA activity (Fig. 8, C and D).

CCS Was Also Able to Inhibit the Production of TNF$\alpha$ and the Phosphorylation of TAK1, JNK/Stressactivated Protein Kinase(SAPK), and p38 in the Mitogen-Activated Protein Kinase Inflammation Signaling Pathway. It is known that TGF- $\beta 1$ and the inflammatory cytokine TNF- $\alpha$ are both stimuli to trigger the mitogen-activated protein kinase (MAPK) inflammatory signaling pathway (Ko et al., 2016). As the expression of TGF- $\beta 1$ was suppressed by CCS (Fig. 5), the downregulation of TNF- $\alpha$ was also investigated in vivo using real-time qPCR and ELISA (Fig. 9). It was clearly demonstrated that CCS also significantly reduced the expression of TNF- $\alpha$ mRNA and protein compared with CS, CL, and CC (Fig. 9).

The activity of classic kinases in the MAPK signaling pathway, such as TAK1, JNK, and P38 mitogen-activated protein kinase (p38), was examined in vivo using ELISA (Fig. 10). Results
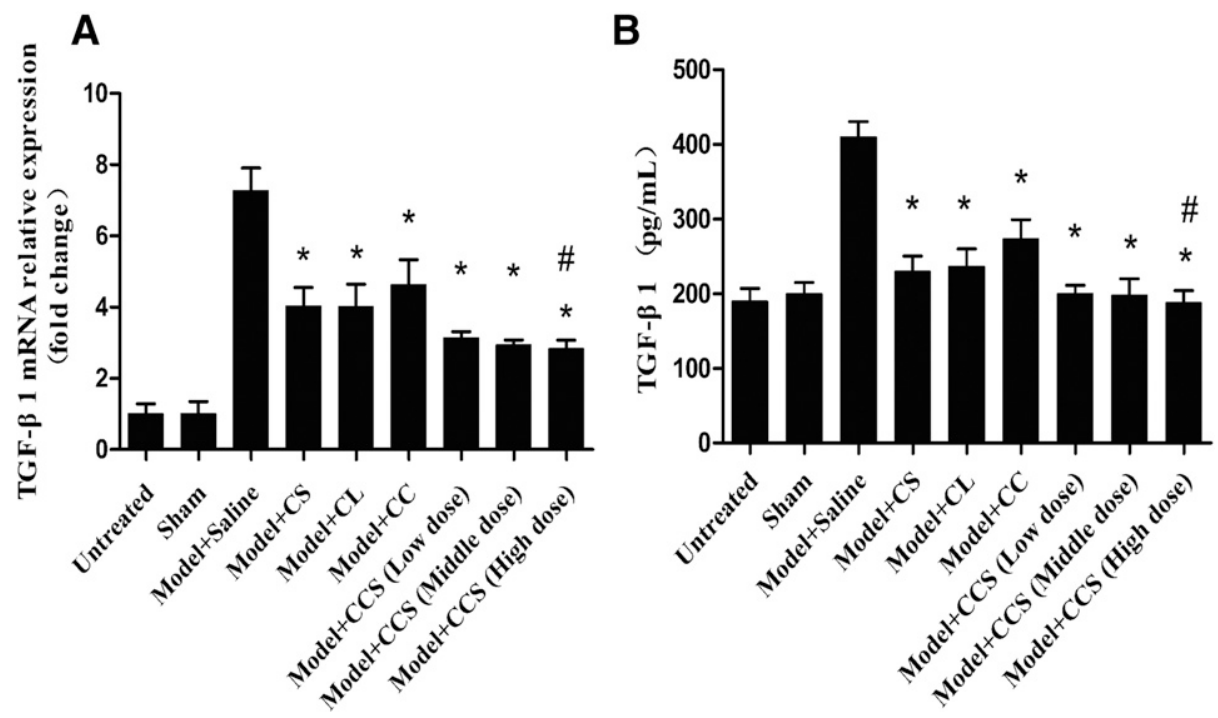

Fig. 5. The expression of TGF- $\beta 1$ mRNA (A) and protein $(\mathrm{B})$ in injured peritoneal tissues. (A) TGF- $\beta 1$ mRNA level in cecum of rats was assessed using real-time qPCR. Compared with the model group (saline), the expression of TGF- $\beta 1$ mRNA in CS, CL, and CC was significantly decreased $(P<0.05)$. In addition, the TGF- $\beta 1$ mRNA level in CCS at high dose was significantly suppressed compared with the $\mathrm{CS}, \mathrm{CL}$, and $\mathrm{CC}$ groups $(P<0.05)$. (B) TGF- $\beta 1$ protein level in serum was evaluated using ELISA. The level of TGF- $\beta 1$ protein in CCS at high dose was also significantly suppressed compared with CS, CL, and CC. Values are presented as the mean \pm S.D. from six animals in each group. ${ }^{\#} P<0.05$, compared with $\mathrm{CS}$, CL, and $\mathrm{CC} ; * P<0.05$, compared with the model group (saline). 


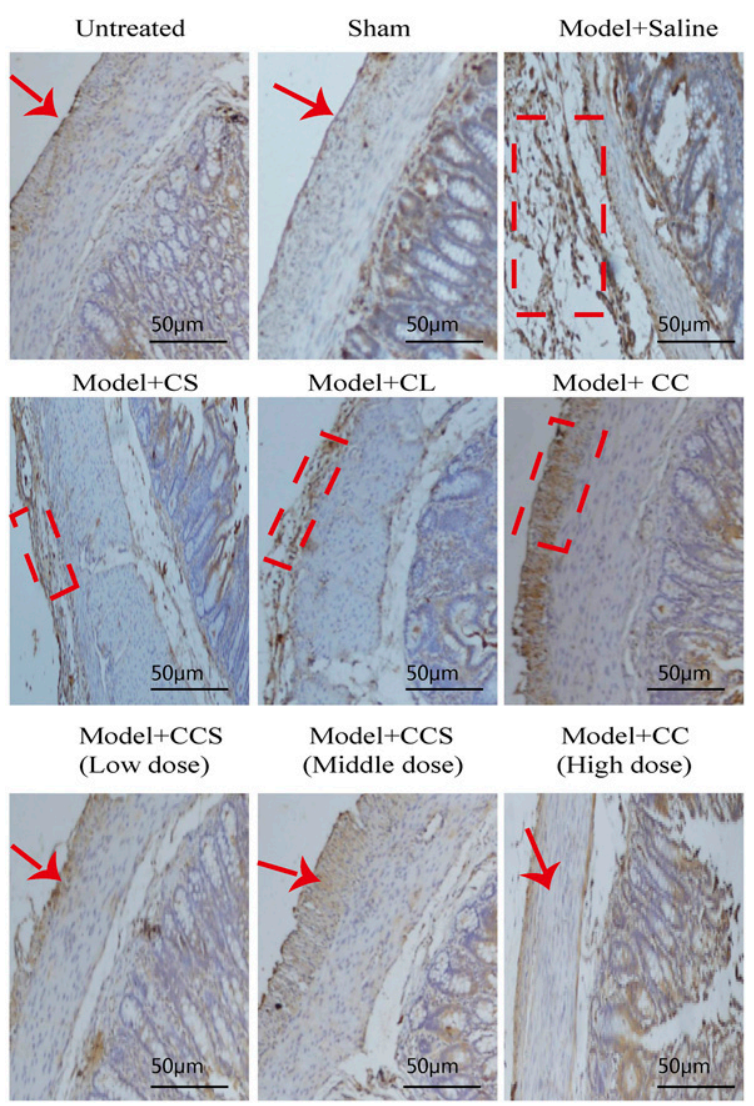

Fig. 6. The expression of TGF- $\beta 1$ was demonstrated using immunohistochemical staining in tissues with adhesion $(n=4)$ (original magnification, $200 \times$; scale bar, $50 \mu \mathrm{m}$ ).

from Fig. 10, A, B, D, and F showed that the expression of TAK1, JNK, and p38 was not affected following treatments. However, as TGF- $\beta 1$ and TNF- $\alpha$ were both suppressed, it was not surprising to note that the activity (phosphorylation) of TAK1, JNK, and p38 was significantly impaired by CCS in three doses, and CCS demonstrated significantly less induction of these kinases compared with CS, CL, and CC (Fig. 10, A, C, E, and $\mathrm{G}$ ).

Taken together, the prevention efficacy of CCS (particularly at high dose) on intra-abdominal adhesion in rats (Fig. 1; Table 1) was mainly due to two aspects: 1) antifibrosis effects -CCS presented a significantly higher inhibitory effect on the expression of TGF- $\beta 1, \alpha$-SMA, and PAI-1 (Figs. 5, 7 and 8, A and $\mathrm{B}$ ), and therefore, achieved significantly better tPA activity (Fig. 8, C and D); and 2) anti-inflammatory effectsCCS could significantly suppress the MAPK signaling pathway (Figs. 9 and 10). These results indicated the clinical potential of CCS in the treatment of postoperative intraabdominal adhesion.

\section{Discussion}

It is known that many natural materials are widely used in biomedical applications, as they have biocompatible, degradable, and nonimmunogenic properties. For instance, CS and its derivatives have been investigated as antiadhesion materials due to the bifunction of separating tissue surfaces during the healing process and modulating the function of inflammation cells (Zhu et al., 2015). In addition, modified CLs have been reported to gel after being placed at the injured sites, form the barriers to separate the traumatized tissue surfaces, and dissolve in the body once the tissues heal (Shao et al., 2017). Furthermore, SPs as an adjuvant material have been applied for wound healing (Sanjeewa et al., 2016). Therefore, a combination of CS, CL, and SP (CCS) was developed with a hope of facilitating both antifibrosis and anti-inflammatory effects on intra-abdominal adhesion in rats (Supplemental Fig. 11).

Results of Fig. 1 and Table 1 confirmed that the adhesion formation between the injured surface and peritoneum in rats was significantly inhibited following the treatment of CCS at a high dose at the time of surgery. CC, a derivative of chitosan created by carboxymethylation reaction, retains the therapeutic effects of chitosan and has been used as a biologic barrier/anti-inflammatory agent to prevent the adhesion
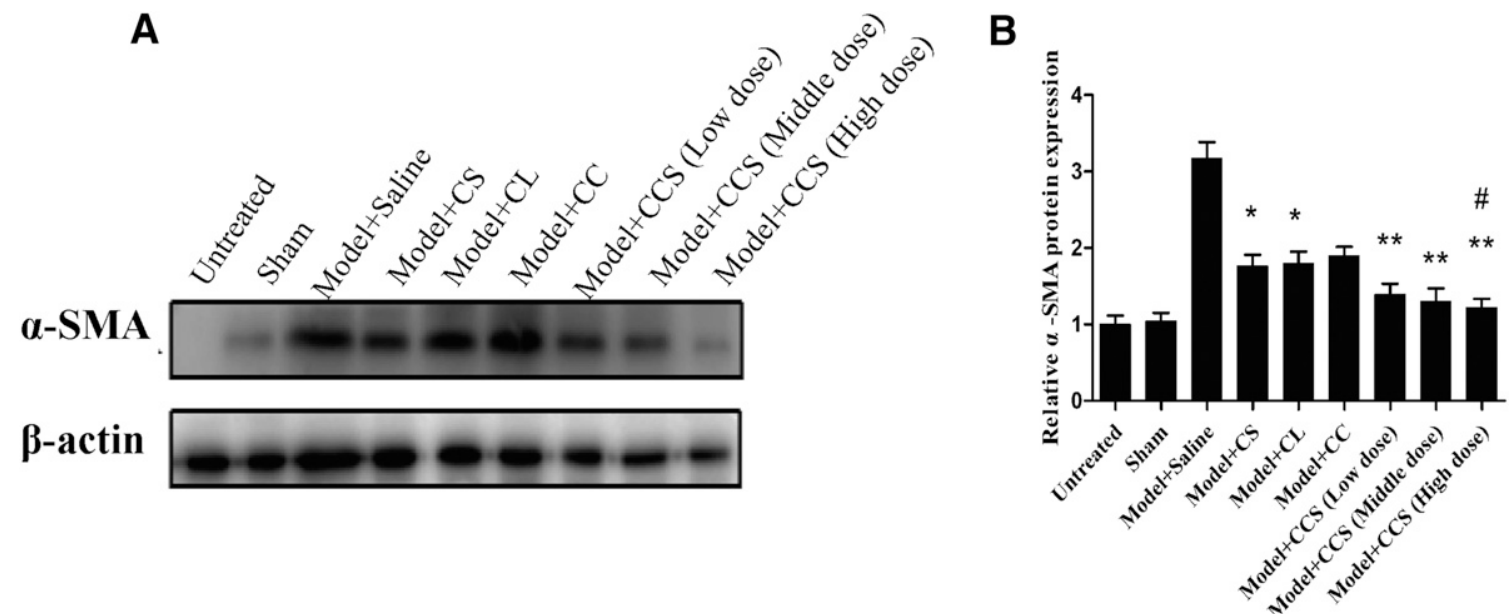

Fig. 7. The expression of $\alpha$-SMA protein was assessed using Western blot. (A) A representative image showed that CCS at three doses effectively reduced the expression of $\alpha$-SMA protein. (B) In addition, CCS at high dose significantly downregulated the $\alpha$-SMA protein compared with CS, CL, and $\mathrm{CC}$ (mean \pm S.D. from four animals in nine groups). ${ }^{\#} P<0.05$, compared with CS, CL, and CC; $* P<0.05$, compared with the model group (saline); ${ }^{* *} P<$ 0.05 , compared with the model group (saline). 


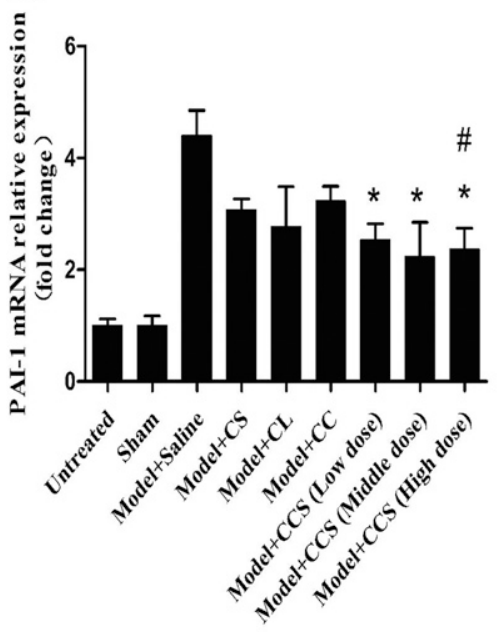

C

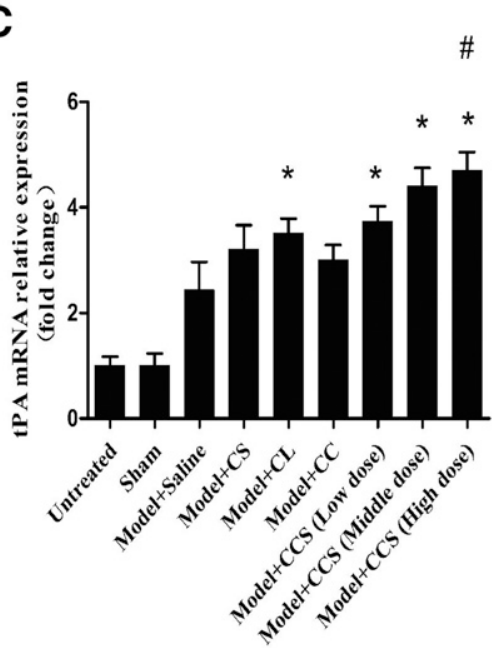

B

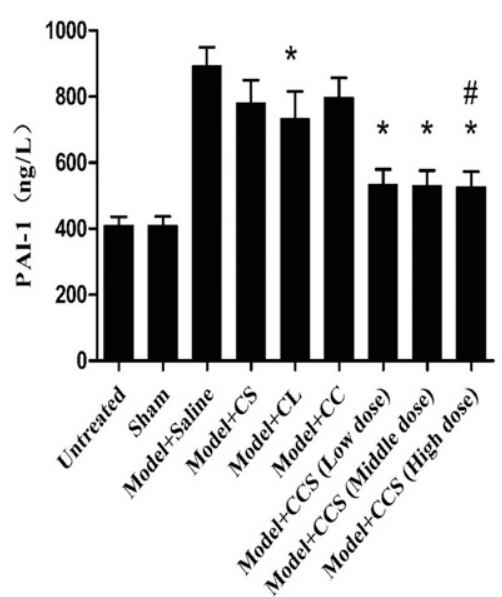

D

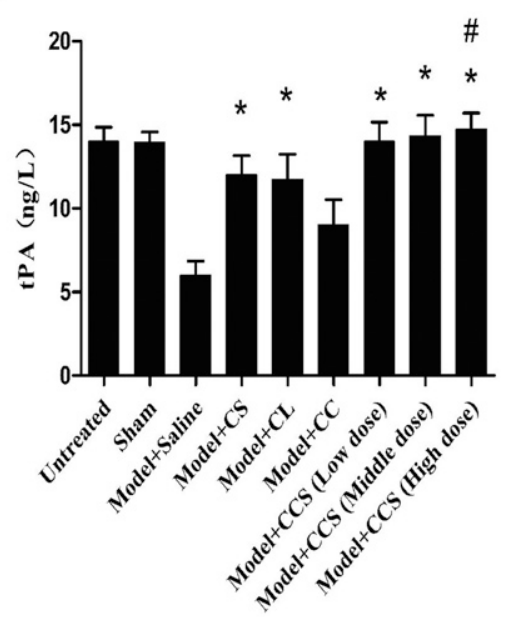

Fig. 8. The mRNA and protein levels of PAI-1 and tPA in injured peritoneal tissues were assessed using real-time qPCR and ELISA. The expression of PAI-1 mRNA (A) and protein (B) were effectively suppressed with CCS at three doses. Following the downregulation of PAI-1, the expression of tPA mRNA (C) and protein (D) was significantly increased with CCS (high dose) compared with CS, CL, and CC. Values are shown as the mean \pm S.D. $(n=6)$. " $P<0.05$, compared with CS, CL, and CC; $* P<0.05$, compared with the model group (saline).
(Daroz et al., 2008). It was interesting to note that CCS at three doses presented significantly better antiadhesion effects in comparison with $\mathrm{CC}$ (considered as a positive control in this study) (Fig. 1; Table 1). The reason that CC did not reduce the adhesion formation as expected is most likely due to the experimental conditions used in this study. In addition, the inhibitory effect achieved by CCS at a high dose was also significantly greater than those obtained by CS and CL (Fig. 1; Table 1) [SP was not used here as a single treatment, as the antiadhesion effects of this compound alone were mild
A

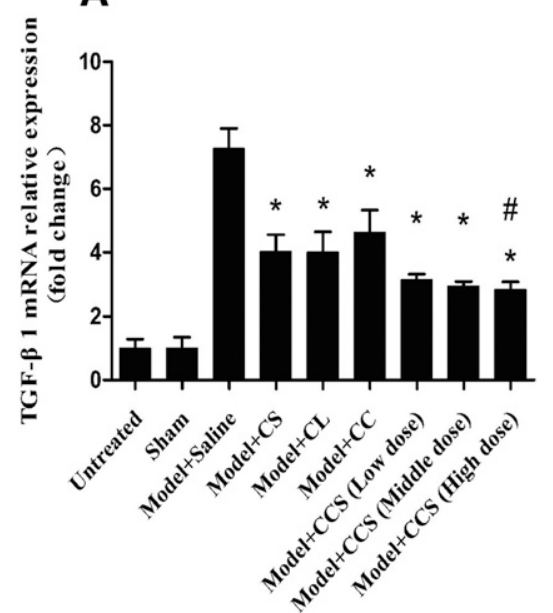

B

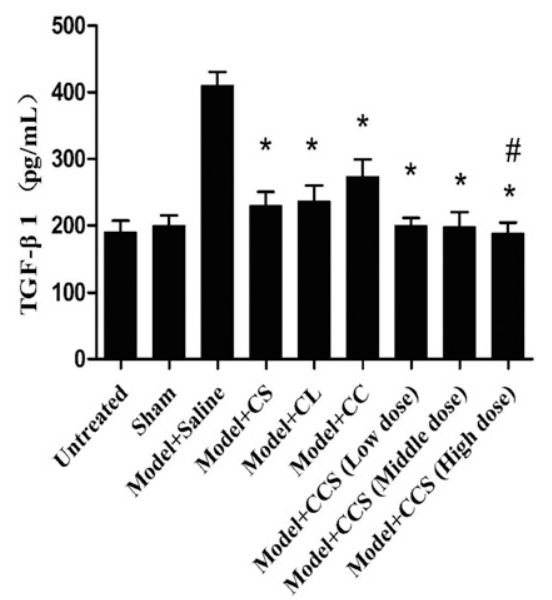

Fig. 9. The mRNA and protein levels of inflammatory cytokine TNF- $\alpha$ in serum were assessed using real-time qPCR (A) and ELISA (B), respectively. The results are presented as the mean \pm S.D. $(n=6)$. ${ }^{\#} P<0.05$, compared with CS, CL, and CC; $* P<0.05$, compared with the model group (saline). 


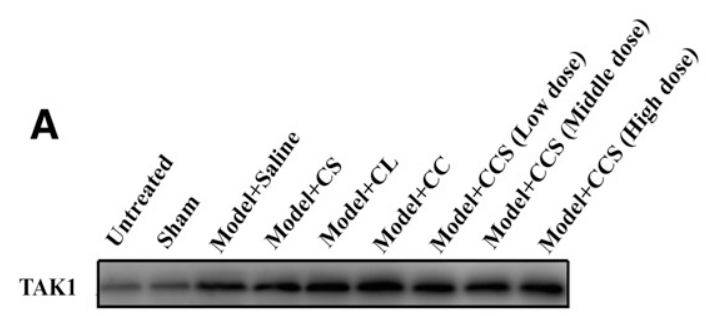

p-TAK1 $-\cdots-\cdots$
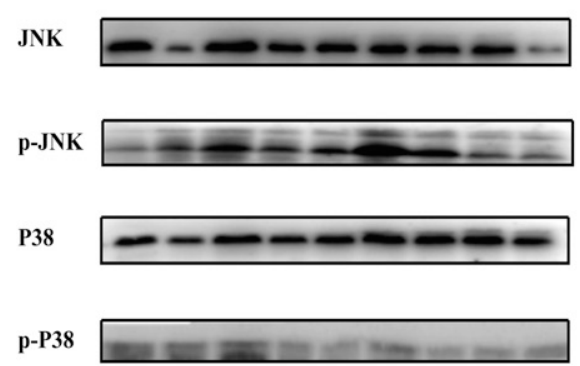

$\beta$-actin

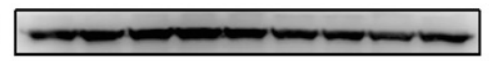

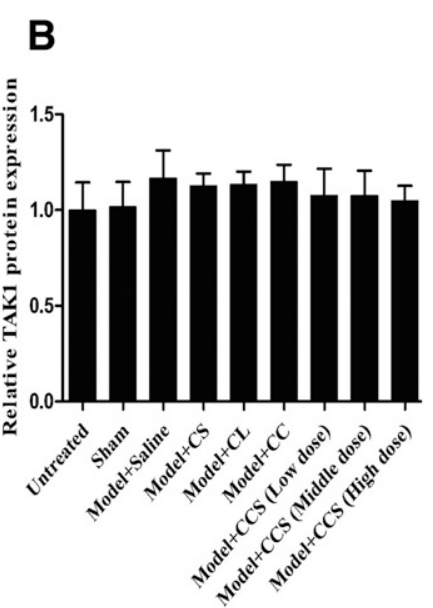
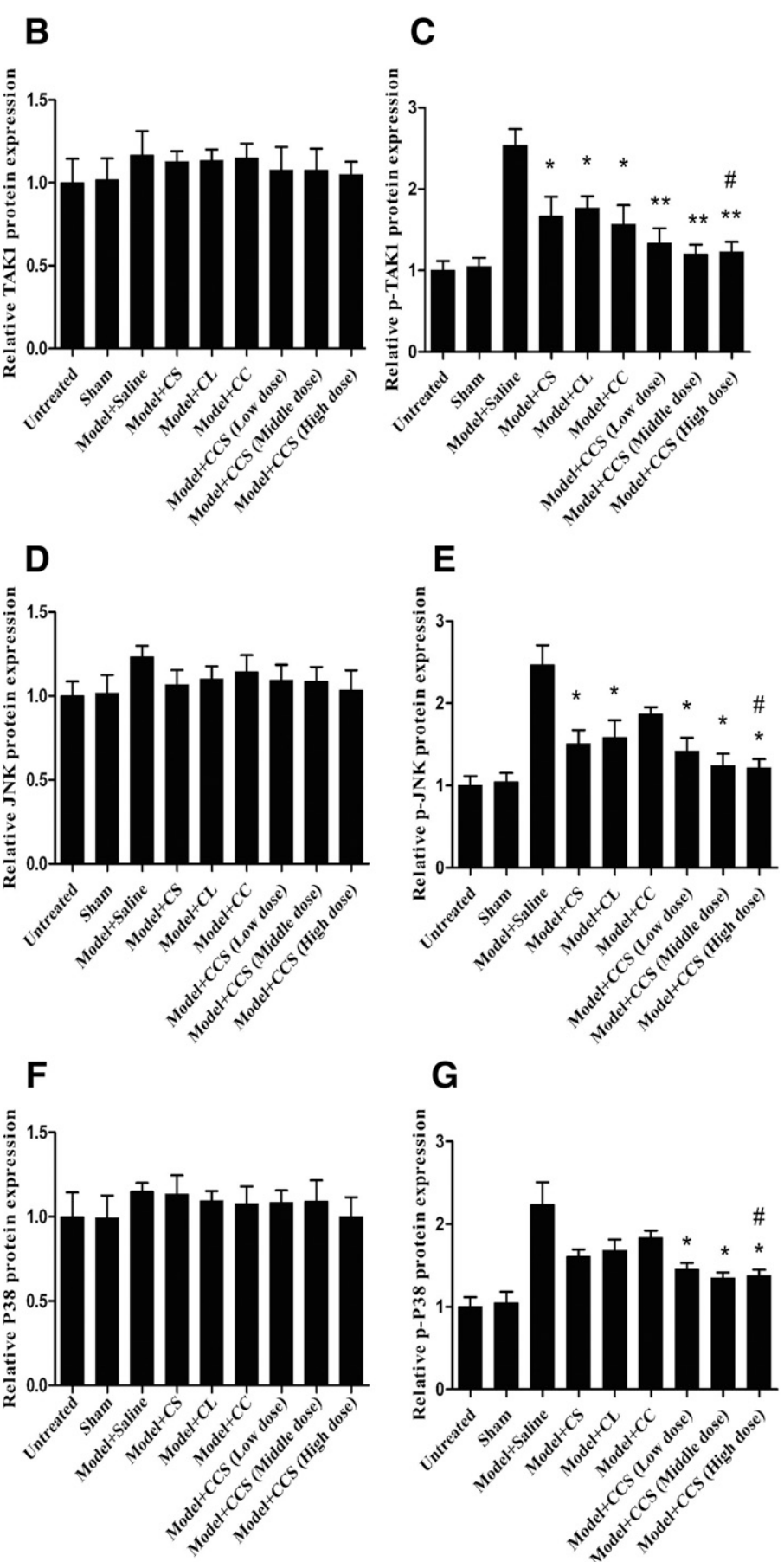

Fig. 10. (A) The activity of TAK1, JNK, and $\mathrm{p} 38$ in intra-abdominal adhesions was confirmed using Western blot. The expression of TAK1 (B), JNK (D), and p38 (F) was not significantly varied between the treatments. However, the phosphorylation of TAK1 (C), JNK (E), and p38 (G) was significantly inactivated using CCS. ${ }^{*} P<0.05$, compared with CS, CL, and CC; ${ }^{*} P<0.05$, compared with the model group (saline); **P<0.05, compared with the model group (saline). Mean \pm S.D. $(n=4)$.

(Chaturvedi et al., 2014)]. These results suggested that CCS was able to effectively prevent intra-abdominal adhesion formation in rats.

The mechanistic studies of CCS as the barrier in prevention of peritoneal adhesion were first investigated using surgical rats. It is known that TGF- $\beta 1$, as the main factor to promote tissue fibrosis and adhesion formation, may synthesize the fibronectin and proteoglycan for the production of collagen and extracellular matrix (ECM) (Wei et al., 2015; Ko et al., 2016).
It has been reported that the level of TGF- $\beta 1$ in adhesion tissues was significantly higher than that in normal peritoneal tissues (Chang et al., 2000; Holmdahl et al., 2001), and downregulation of TGF- $\beta 1$ reduced the formation of peritoneal adhesions (Zheng et al., 2013). In this study, the expression of TGF- $\beta 1$ mRNA and protein was significantly inhibited by CCS in vivo when compared with $\mathrm{CS}, \mathrm{CL}$, and $\mathrm{CC}$ (Fig. 5). Immunohistochemical observation also showed that CCS achieved downregulation of TGF- $\beta 1$ (Fig. 6). One of TGF- $\beta 1$ 
downstream factors, $\alpha$-SMA, is an important fibroblast biomarker often identified in the injured tissues (Wei et al., 2017). Western blot results in Fig. 7 showed that the $\alpha$-SMA protein was also effectively downregulated in three CCS groups.

It is known that the deposition of fibrin is induced by PAI-1 (one of the TGF- $\beta 1$ downstream factors), causing the adhesion formation (Aarons et al., 2007; Esposito et al., 2013). It has been reported that the downregulation of the PAI-1 gene mediated by RNA interference in rats could prevent the abdominal adhesion (Chegini et al., 2001). In this study, the high expression of PAI-1 was found in the model group, and in contrast, levels of PAI-1 mRNA and protein were dramatically reduced by CCS at various doses (Fig. 8, A and B), supporting antiadhesion effects as observed in the results of Fig. 1 and Table 1.

As tPA is negatively regulated by PAI-1, downregulation of PAI-1 may enhance the expression of tPA (Yang et al., 2008; Fotiadis et al., 2015; Honjo et al., 2017). Indeed, the levels of tPA mRNA and protein were significantly increased following CCS treatments (Fig. 8, C and D), which was most likely to due to the suppression of PAI-1 (Fig. 8, A and B). It is also known that the degradation of fibrin is improved by plasmin (a fibrinolytic factor, which can break down collagen fibers) (Cassidy et al., 2014), and the generation of plasmin was enhanced by tPA (Reed et al., 2004). As shown in Fig. 1 and Table 1, the intra-abdominal adhesions were significantly reduced in surgical rats treated using CCS at three doses. Therefore, these results suggested that CCS, as the antiadhesion barrier, could effectively inhibit the postoperative intra-abdominal adhesion in rats partly through blocking the TGF- $\beta 1$-associated pathway and resulting in the degradation of collagen fibers at injured sites (Supplemental Fig. 11).

The mechanistic studies of CCS as the anti-inflammatory drug in prevention of peritoneal adhesion were next assessed using surgical rats. It is known that at the early stage after peritoneal injury, inflammatory responses occur at traumatized sites, in which various proinflammatory factors (e.g., TGF- $\beta 1$, TNF- $\alpha$, IL-1 $\beta$, and IL-6) are released (Zhang et al., 2014). The MAPK inflammation signaling pathway has been reported to be activated by these proinflammatory cascades (Wang et al., 2017). As TGF- $\beta 1$ and TNF- $\alpha$ were effectively suppressed by CCS (Figs. 5 and 9), it was not surprising to note that the activity of classic kinases in the MAPK signaling pathway, such as TAK1, JNK, and p38, was also inhibited (Fig. 10). It is also known that phosphorylation of TAK1 may act as the stimulus to induce the phosphorylation of JNK and p38, as a result of the transcription regulation of inflammation mediators and proinflammatory cytokines to promote systemic inflammation (Ayroldi et al., 2012). These results suggest that CCS, as the anti-inflammation drug, was able to inhibit inflammatory responses via downregulation of the MAPK signaling pathway, resulting in prevention of intraabdominal adhesion (Supplemental Fig. 11).

It is interesting to note that CCS at high dose achieved significantly better prevention results compared with CCS at low and middle doses (Fig. 1; Table 1). However, mechanistic studies of CCS as the antiadhesion barrier demonstrated that the downregulation of TGF- $\beta 1, \alpha$-SMA, and PAI- 1 and the activation of tPA achieved by CCS at high dose were not significantly better than those obtained by CCS at low and middle doses (Figs. 5, 7, and 8). It was most likely that the PAI1 expression can be regulated by different TGF- $\beta 1$-associated signaling pathways, such as the Smads/p53 pathway (Kawarada et al., 2016), Mitogen-activated Extracellular signal-regulated Kinase (MEK)/ERK pathway (Samarakoon et al., 2005), and Yes-associated protein/transcriptional coactivator with PDZ-binding motif pathway (Thomasy et al., 2013). In addition, the remodeling of the ECM that causes fibrotic tissue or scar due to collagen deposition in the skin has been found for wound healing (Philips et al., 2004). It is also known that metalloproteinases (a family of proteases) are essential to the ECM remodeling process (Philips et al., 2004). It has recently been reported that TGF- $\beta 1$ was able to impair the interaction of metalloproteinases with their inhibitors (tissue inhibitor of matrix metalloproteinases), resulting in production of the ECM in injured tissues (Philips et al., 2004). Therefore, the influence of CCS on the cross-talk of these TGF$\beta$-associated signaling pathways, to improve the understanding of antiadhesion effects (i.e., downregulation of fibrosis and promotion of fibrolysis), will be the focus of future work.

Similarly, mechanistic studies of CCS as the antiinflammatory drug showed that CCS at high dose did not achieve significantly better reduction of TAK-1, JNK, and p38 cascades compared with CCS at low and middle doses (Fig. 10). It has been reported that the activation of the MAPK pathway can also be regulated by IL- $1 \beta$ and IL-6, causing inflammation within injured tissues (Wang et al., 2017). In addition to the MAPK pathway, inflammatory pathways such as Nuclear Factor-к-gene Binding(NF-кB) (Tsai et al., 2013), MEK/ERK (Samarakoon et al., 2005), and Ras homologue protein/Rho associated coiled coil forming protein (Rao et al., 2017) are associated with the formation of intra-abdominal adhesion (Supplemental Fig. 11). Therefore, the influence of CCS on these signaling cascades, with a hope to improve the understanding of anti-inflammation effects, will be investigated in the future.

Prevention of postoperative intra-abdominal adhesion mainly resulted from the downregulation of fibrosis, promotion of fibrolysis, and inhibition of inflammatory response (Ambler et al., 2012) In this study, a combined prevention strategy using the coadministration of three natural materials (chitosan, cellulose, and seaweed polysaccharide) resulted in additive antiadhesion effects in rats with abdominal surgery. The resultant adhesion prevention was due to both antifibroblastic and anti-inflammatory effects. In addition, CCS did not cause any significant toxicity, as the animal body weights treated with CCS were similar to those of the untreated and sham groups (data not shown). As a result, CCS holds great therapeutic potential in the treatment of postoperative intraabdominal adhesion.

\section{Authorship Contributions}

Participated in research design: Tian, Pei.

Conducted experiments: Tian, H. Li, Yan Li, Liu, Sun, Z. Cong, Luan, L. Wang, Ren, D. Cong, H. Wang.

Performed data analysis: Tian, Yao Li, Chen.

Wrote or contributed to the writing of the manuscript: L. Tian, H. Li.

\section{References}

Aarons CB, Cohen PA, Gower A, Reed KL, Leeman SE, Stucchi AF, and Becker JM (2007) Statins (HMG-CoA reductase inhibitors) decrease postoperative adhesions by increasing peritoneal fibrinolytic activity. Ann Surg 245:176-184.

Ambler DR, Fletcher NM, Diamond MP, and Saed GM (2012) Effects of hypoxia on the expression of inflammatory markers IL-6 and TNF-a in human normal peritoneal and adhesion fibroblasts. Syst Biol Reprod Med 58:324-329.

Ayroldi E, Cannarile L, Migliorati G, Nocentini G, Delfino DV, and Riccardi C (2012) Mechanisms of the anti-inflammatory effects of glucocorticoids: genomic and nongenomic interference with MAPK signaling pathways. FASEB J 26:4805-4820. 
Bi J, Zhang S, Du Z, Zhang J, Deng Y, Liu C, and Zhang J (2017) Peripheral serotonin regulates postoperative intra-abdominal adhesion formation in mice. Sci Rep $\mathbf{7}$ : 10001.

Cassidy MR, Sherburne AC, Sheldon HK, Gainsbury ML, Heydrick S, and Stucchi AF (2014) Histone deacetylase inhibitors decrease intra-abdominal adhesions with one intraoperative dose by reducing peritoneal fibrin deposition pathways. Surgery 155:234-244

Chang J, Thunder R, Most D, Longaker MT, and Lineaweaver WC (2000) Studies in flexor tendon wound healing: neutralizing antibody to TGF-beta1 increases postoperative range of motion. Plast Reconstr Surg 105:148-155.

Chaturvedi AA, Lomme RM, Hendriks T, and van Goor H (2014) Ultrapure alginate anti-adhesion gel does not impair colon anastomotic strength. J Surg Res 192: 432-439.

Chegini N, Kotseos K, Zhao Y, Bennett B, McLean FW, Diamond MP, Holmdahl L, and Burns J (2001) Differential expression of TGF-beta1 and TGF-beta3 in serosal tissues of human intraperitoneal organs and peritoneal adhesions. Hum Reprod 16:1291-1300

Daroz LR, Lopes JB, Dallan LA, Campana-Filho SP, Moreira LF, and Stolf NA (2008) Prevention of postoperative pericardial adhesions using thermal sterile carboxymethyl chitosan. Rev Bras Cir Cardiovasc 23:480-487.

Deng L, Li Q, Lin G, Huang D, Zeng X, Wang X, Li P, Jin X, Zhang H, Li C, et al. (2016) P-glycoprotein mediates postoperative peritoneal adhesion formation by enhancing phosphorylation of the chloride channel-3. Theranostics 6: $204-218$

Esposito AJ, Heydrick SJ, Cassidy MR, Gallant J, Stucchi AF, and Becker JM (2013) Substance $\mathrm{P}$ is an early mediator of peritoneal fibrinolytic pathway genes and promotes intra-abdominal adhesion formation. J Surg Res 181:25-31.

Fotiadis K, Filidou E, Arvanitidis K, Valatas V, Stavrou G, Basdanis G, Paspaliaris V, Kolios G, and Kotzampassi K (2015) Intraperitoneal application of phospholipids for the prevention of postoperative adhesions: a possible role of myofibroblasts. J Surg Res 197:291-300.

Francesko A and Tzanov T (2011) Chitin, chitosan and derivatives for wound healing and tissue engineering. Adv Biochem Eng Biotechnol 125:1-27.

Gomel V, Urman B, and Gurgan T (1996) Pathophysiology of adhesion formation and strategies for prevention. J Reprod Med 41:35-41.

Guo J, Ogier JR, Desgranges S, Darcy R, and O'Driscoll C (2012) Anisamide-targeted cyclodextrin nanoparticles for siRNA delivery to prostate tumours in mice. Biomaterials 33:7775-7784.

Guo J, Russell EG, Darcy R, Cotter TG, McKenna SL, Cahill MR, and O'Driscoll CM (2017) Antibody-targeted cyclodextrin-based nanoparticles for siRNA delivery in the treatment of acute myeloid leukemia: physicochemical characteristics, in vitro mechanistic studies, and ex vivo patient derived therapeutic efficacy. Mol Pharm 14:940-952.

Holmdahl L, Kotseos K, Bergström M, Falk P, Ivarsson ML, and Chegini N (2001) Overproduction of transforming growth factor-beta1 (TGF-beta1) is associated with adhesion formation and peritoneal fibrinolytic impairment. Surgery 129:626-632.

Honjo K, Munakata S, Tashiro Y, Salama Y, Shimazu H, Eiamboonsert S, Dhahri D, Ichimura A, Dan T, Miyata T, et al. (2017) Plasminogen activator inhibitor-1 regulates macrophage-dependent postoperative adhesion by enhancing EGF-HER1 signaling in mice. FASEB J 31:2625-2637.

Hu J, Fan D, Lin X, Wu X, He X, He X, Wu X, and Lan P (2015) Safety and efficacy of sodium hyaluronate gel and chitosan in preventing postoperative peristomal ad hesions after defunctioning enterostomy: a prospective randomized controlled trials. Medicine (Baltimore) 94:e2354

Kawarada Y, Inoue Y, Kawasaki F, Fukuura K, Sato K, Tanaka T, Itoh Y, and Hayashi H (2016) TGF- $\beta$ induces p53/Smads complex formation in the PAI-1 promoter to activate transcription. Sci Rep 6:35483.

Ko W, Sohn JH, Jang JH, Ahn JS, Kang DG, Lee HS, Kim JS, Kim YC, and Oh H (2016) Inhibitory effects of alternaramide on inflammatory mediator expression through TLR4-MyD88-mediated inhibition of NF-kB and MAPK pathway signaling in lipopolysaccharide-stimulated RAW264.7 and BV2 cells. Chem Biol Interact 244 $16-26$.

Liakakos T, Thomakos N, Fine PM, Dervenis C, and Young RL (2001) Peritoneal adhesions: etiology, pathophysiology, and clinical significance. Recent advances in prevention and management. Dig Surg 18:260-273.

Lou W, Zhang H, Ma J, Zhang D, Liu C, Wang S, Deng Z, Xu H, and Liu J (2012) In vivo evaluation of in situ polysaccharide based hydrogel for prevention of postoperative adhesion. Carbohydr Polym 90:1024-1031.

Nair SK, Bhat IK, and Aurora AL (1974) Role of proteolytic enzyme in the prevention of postoperative intraperitoneal adhesions. Arch Surg 108:849-853.
Parsak CK, Satar S, Akcam T, Satar D, and Sungur I (2007) Effectiveness of treatment to prevent adhesions after abdominal surgery: an experimental evaluation in rats. Adv Ther 24:796-802.

Philips N, Keller T, and Gonzalez S (2004) TGF beta-like regulation of matrix metalloproteinases by anti-transforming growth factor-beta, and anti-transforming growth factor-beta 1 antibodies in dermal fibroblasts: implications for wound healing. Wound Repair Regen 12:53-59.

Poehnert D, Grethe L, Maegel L, Jonigk D, Lippmann T, Kaltenborn A, Schrem H, Klempnauer J, and Winny M (2016) Evaluation of the effectiveness of peritoneal adhesion prevention devices in a rat model. Int $J$ Med Sci 13:524-532.

Rao J, Ye Z, Tang H, Wang C, Peng H, Lai W, Li Y, Huang W, and Lou T (2017) The RhoA/ROCK pathway ameliorates adhesion and inflammatory infiltration induced by AGEs in glomerular endothelial cells. Sci Rep 7:39727.

Reed KL, Fruin AB, Gower AC, Stucchi AF, Leeman SE, and Becker JM (2004) A neurokinin 1 receptor antagonist decreases postoperative peritoneal adhesion formation and increases peritoneal fibrinolytic activity. Proc Natl Acad Sci USA 101:9115-9120.

Risberg B (1997) Adhesions: preventive strategies. Eur J Surg Suppl (577):32-39. Rocha HA, Franco CR, Trindade ES, Carvalho LC, Veiga SS, Leite EL, Dietrich CP and Nader HB (2001) A fucan from the brown seaweed Spatoglossum schröeder inhibits Chinese hamster ovary cell adhesion to several extracellular matrix proteins. Braz J Med Biol Res 34:621-626.

Ryan CK and Sax HC (1995) Evaluation of a carboxymethylcellulose sponge for prevention of postoperative adhesions. Am J Surg 169:154-159, discussion $159-160$.

Samarakoon R, Higgins CE, Higgins SP, Kutz SM, and Higgins PJ (2005) Plasminogen activator inhibitor type-1 gene expression and induced migration in TGFbeta1-stimulated smooth muscle cells is pp60(c-src)/MEK-dependent. J Cell Physiol 204:236-246.

Sanjeewa KKA, Kim EA, Son KT, and Jeon YJ (2016) Bioactive properties and potentials cosmeceutical applications of phlorotannins isolated from brown seaweeds: a review. J Photochem Photobiol B 162:100-105.

Shao W, Wu J, Liu H, Ye S, Jiang L, and Liu X (2017) Novel bioactive surface functionalization of bacterial cellulose membrane Carbohydr Polym 178.270-276.

Thomasy SM, Morgan JT, Wood JA, Murphy CJ, and Russell P (2013) Substratum stiffness and latrunculin $\mathrm{B}$ modulate the gene expression of the mechanotransducers YAP and TAZ in human trabecular meshwork cells. Exp Eye Res 113:66-73.

Tsai SH, Huang PH, Peng YJ, Chang WC, Tsai HY, Leu HB, Chen JW, and Lin SJ (2013) Zoledronate attenuates angiotensin II-induced abdominal aortic aneurysm through inactivation of Rho/ROCK-dependent JNK and NF-кB pathway. Cardiovasc Res 100:501-510.

Wang K, Wang F, Bao JP, Xie ZY, Chen L, Zhou BY, Xie XH, and Wu XT (2017) Tumor necrosis factor $\alpha$ modulates sodium-activated potassium channel SLICK in rat dorsal horn neurons via p38 MAPK activation pathway. J Pain Res 10: $1265-1271$

Wei G, Chen X, Wang G, Jia P, Xu Q, Ping G, Wang K, and Li X (2015) Inhibition of cyclooxygenase- 2 prevents intra-abdominal adhesions by decreasing activity of peritoneal fibroblasts. Drug Des Devel Ther 9:3083-3098.

Wei G, Wu Y, Gao Q, Zhou C, Wang K, Shen C, Wang G, Wang K, Sun X, and Li X (2017) Effect of emodin on preventing postoperative intra-abdominal adhesion formation. Oxid Med Cell Longev 2017:1740317.

Yang B, Gong C, Zhao X, Zhou S, Li Z, Qi X, Zhong Q, Luo F, and Qian Z (2012) Preventing postoperative abdominal adhesions in a rat model with PEG-PCL-PEG hydrogel. Int $J$ Nanomedicine 7:547-557.

Yang Y, Yang S, Chen M, Zhang X, Zou Y, and Zhang X (2008) Compound Astragalus and Salvia miltiorrhiza extract exerts anti-fibrosis by mediating TGF-beta/Smad signaling in myofibroblasts. J Ethnopharmacol 118:264-270.

Zhang Y, Li X, Zhang Q, Li J, Ju J, Du N, Liu X, Chen X, Cheng F, Yang L, et al. (2014) Berberine hydrochloride prevents postsurgery intestinal adhesion and inflammation in rats. $J$ Pharmacol Exp Ther 349:417-426.

Zheng Z, Zhang W, Sun W, Li X, Duan J, Cui J, Feng Z, and Mansour HM (2013) Influence of the carboxymethyl chitosan anti-adhesion solution on the TGF- $\beta 1$ in a postoperative peritoneal adhesion rat. J Mater Sci Mater Med 24:2549-2559.

Zhu L, Peng L, and Zhang YQ (2015) The processing of chitosan and its derivatives and their application for postoperative anti-adhesion. Mini Rev Med Chem 15:330-337.

Address correspondence to: Jin Pei, School of Pharmaceutical Sciences, Jilin University, Changchun, Jilin 130000, China. E-mail: peijin@jlu.edu.cn 